\title{
Atmospheric neutrino oscillations with IceCube
}

\author{
Andrii Terliuk* for the IceCube collaboration ${ }^{\dagger}$ \\ Deutsches Elektronen-Synchrotron, Platanenallee 6, 15738 Zeuthen, Germany \\ E-mail: andrii.terliuk@desy.de
}

\begin{abstract}
The IceCube Neutrino Observatory is a cubic-kilometer Cherenkov neutrino detector located in the glacial ice at the geographic South Pole. DeepCore, a denser sub-array within the detector, has a neutrino detection energy threshold of less than $10 \mathrm{GeV}$. In recent years, IceCube opened a new window for measurements of the atmospheric mixing parameters in ice and became one of the leading instruments measuring atmospheric neutrino oscillations. Furthermore, its data serve as a probe of new physics beyond the standard three-neutrino paradigm and limit the allowed mixing between active and potential sterile neutrino species. This contribution discusses IceCube's most recent measurements of $v_{\mu}$ disappearance and $v_{\tau}$ appearance, searches for sterile neutrino mixing, as well as future in-ice measurements of fundamental neutrino properties.
\end{abstract}

Neutrino Oscillation Workshop (NOW2018)

9 - 16 September, 2018

Rosa Marina (Ostuni, Brindisi, Italy)

\footnotetext{
*Speaker.

${ }^{\dagger}$ https://icecube.wisc.edu/
} 
The IceCube Neutrino Observatory [1] is a cubic-kilometer Cherenkov neutrino detector located at the geographic South Pole. It consists of 5160 digital optical modules (DOMs) grouped in vertical strings deployed in the glacial ice. The high energy part of the in-ice detector consists of 78 such strings with a $125 \mathrm{~m}$ lateral and $17 \mathrm{~m}$ vertical spacing between the modules. It instruments the depth between approximately $1450 \mathrm{~m}$ and $2450 \mathrm{~m}$ below the ice surface. The detector has a neutrino detection energy threshold of approximately $100 \mathrm{GeV}$ and has a primary purpose of studying high energy astrophysical neutrinos.

DeepCore [2] is a denser sub-array placed in the center of the bottom part of the main IceCube array. It consists of 8 strings with the vertical spacing of $7 \mathrm{~m}$ deployed 40-60 m apart from each other. The sub-array instruments the depth between $2100 \mathrm{~m}$ and $2450 \mathrm{~m}$, the region where the glacial ice has the best optical properties with longer optical absorption and scattering lengths. DeepCore has an energy threshold of less than $10 \mathrm{GeV}$ making it a suitable tool for studies of the atmospheric neutrino oscillations. In addition, it benefits from the surrounding IceCube strings that serve as the veto against the atmospheric muon background.

The main effect of atmospheric neutrino oscillations is a transition of $v_{\mu}$ into $v_{\tau}$ described by the probability ${ }^{1}$

$$
P\left(v_{\mu} \rightarrow v_{\tau}\right) \approx \sin ^{2}\left(\theta_{23}\right) \sin ^{2}\left(\Delta m_{32}^{2} \frac{L}{4 E_{v}}\right),
$$

where $\Delta m_{32}^{2}$ and $\theta_{23}$ are the atmospheric neutrino mass splitting and mixing angle, respectively; $E_{v}$ is the neutrino energy, and $L$ is the distance between the neutrino production and detection points. For atmospheric neutrinos, the distance $L$ is given by the neutrino arrival direction. The up-going neutrinos (cosine of the zenith angle $\cos \theta_{Z} \approx-1$ ), the distance is about $12700 \mathrm{~km}$, while for down-going neutrinos $\left(\cos \theta_{Z} \approx 1\right)$ the travelled distance is only about $20 \mathrm{~km}$.

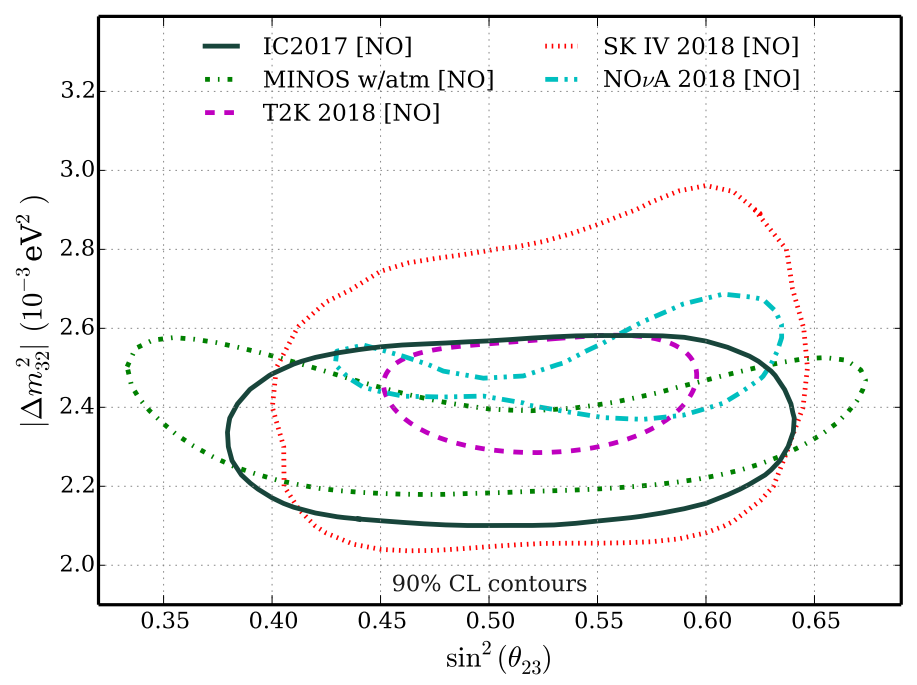

Figure 1: The results of the IceCube measurement of the atmospheric neutrino oscillations compared to the results from other experiments [3].

\footnotetext{
${ }^{1}$ The two-neutrino approximation is given for simplicity here; actual measurements use the full calculation of neutrino oscillation probabilities in matter.
} 
Since the mixing angle $\theta_{23}$ has a value close to $45^{\circ}$, neutrino oscillations result in an almost complete transition of the up-going muon neutrinos with energy of about $25 \mathrm{GeV}$ into tau neutrinos. A precise energy of this $v_{\mu}$ disappearance minimum is given by $\Delta m_{32}^{2}$, while the oscillation amplitude is related to $\theta_{23}$. Measurements of the atmospheric oscillations probe the neutrino oscillation effect at the highest energies possible with terrestrial neutrino sources.

Figure 1 shows the recent IceCube measurement [3] of atmospheric neutrino oscillations in the $v_{\mu}$ disappearance channel. It uses three years of the IceCube data in the reconstructed energy range between $6 \mathrm{GeV}$ and $56 \mathrm{GeV}$ taken in the 2012-2014 seasons. This analysis is done using the full range of zenith angles, resulting in the simultaneous probing of the baselines between approximately $20 \mathrm{~km}$ and $12700 \mathrm{~km}$. This measurement results in the precision for the atmospheric mixing parameters that approach the accuracy of the dedicated accelerator experiments, but performed at higher energies and longer baselines.

A range of anomalies are observed in some of the accelerator, reactor, and radiochemical neutrino experiments. Such anomalies can be interpreted as the result of sterile neutrino mixing beyond the standard three-neutrino paradigm [5]. In this case, the mixing matrix must be nonunitary for the standard three-neutrino mixing. One of the ways to probe it is a measurement of the $v_{\tau}$ appearance given by Equation (1). A normalization of the appeared $v_{\tau}$ flux smaller than 1 with respect to the standard oscillation expectation might indicate the transition of $v_{\mu}$ into the hidden sterile sector, while values larger than 1 can be a sign of more exotic neutrino properties. The results of the two IceCube $v_{\tau}$ appearance measurements [4] are shown in Figure 2. The study marked as Analysis 1 uses a dedicated event selection with approximately 50\% more events than in the muon neutrino disappearance sample used in Analysis 2. The measurements are in good agreement with the standard three-neutrino model and are currently among the most precise measurements of the tau neutrino appearance.

Another way to test the aforementioned anomalies is to search for the sterile neutrino mixing

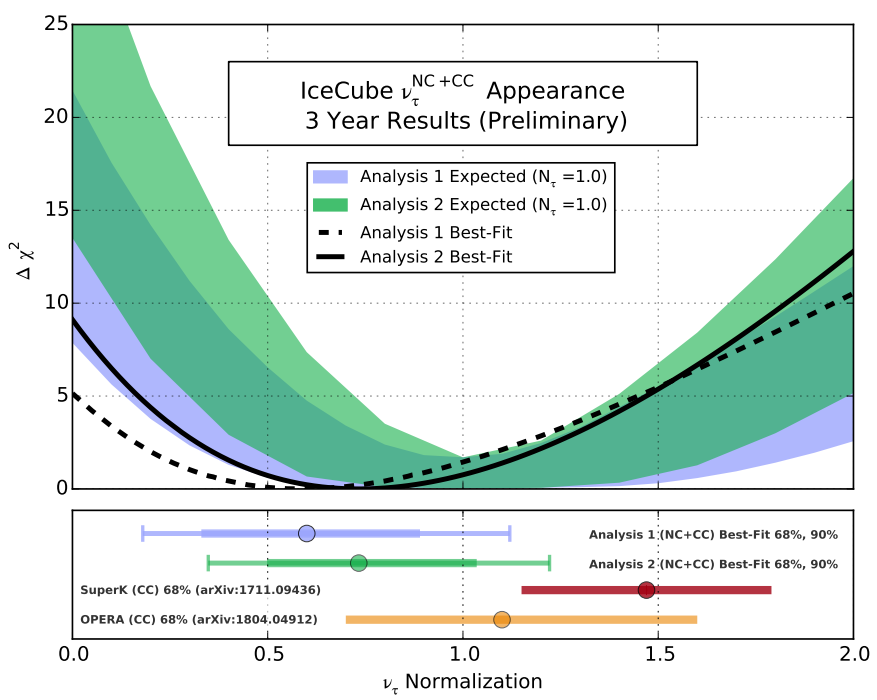

Figure 2: The results of the IceCube $v_{\tau}$ appearance measurements compared to the results from other experiments [4]. 


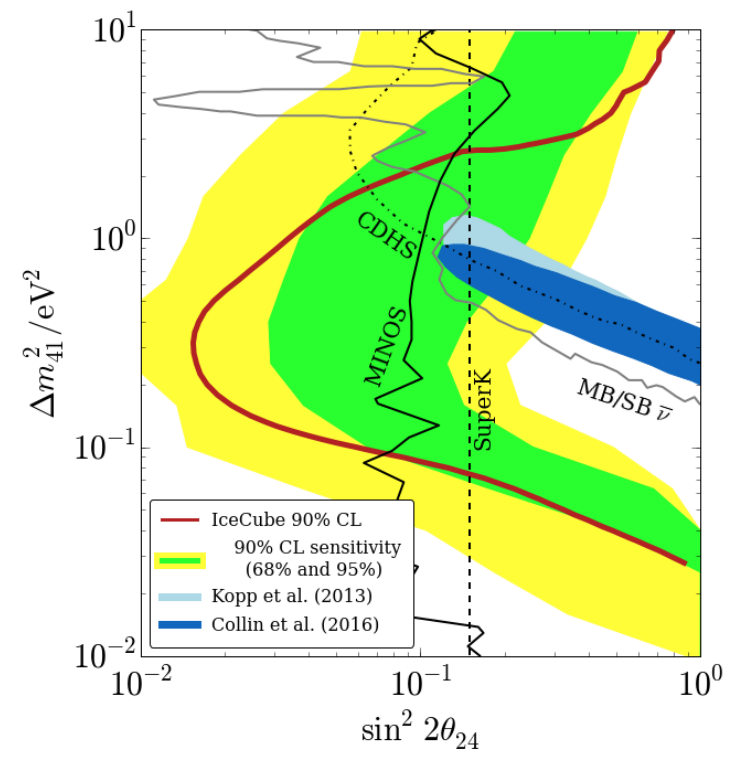

Figure 3: The limit on the allowed sterile neutrino mixing using TeV neutrinos in IceCube [6].

effects directly. IceCube makes a range of searches for sterile neutrinos within the " $3+1$ " model that assumes one heavier sterile neutrino. Figure 3 shows the result of the one-year study [6] probing the mantle-core-mantle resonant enhancement of the $\bar{v}_{\mu}$ transition probability into the sterile neutrino state. Another search [7], shown in Figure 4, tests the matter induced impact of the sterile neutrino mixing on the standard neutrino oscillations governed by Equation (1) using 3 years of DeepCore data. The IceCube searches found no indications of the sterile neutrino mixing and, therefore, place

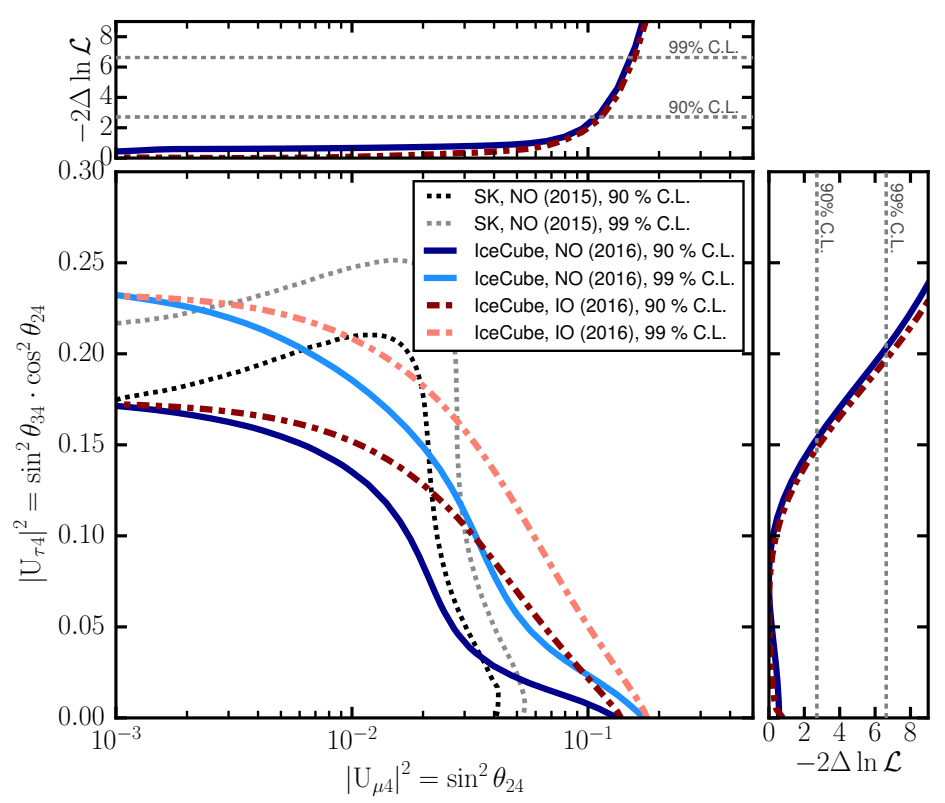

Figure 4: The exclusion contours for the allowed sterile neutrino mixing at $\Delta m_{41}^{2}=1 \mathrm{eV}^{2}$ from the DeepCore low energy search [7]. 
strict limits on the allowed mixing of muon and tau neutrinos with the sterile neutrino state.

In additon to the aforementioned measurements, the IceCube collaboration probed the neutrino mass ordering [8] and placed limits on non-standard neutrino interactions [9]. Currently, several neutrino oscillation studies on the 3 year sample are in preparation as well as the results from up to 7 years of the IceCube data.

In the future, the IceCube Upgrade will provide even more precision to the measurement of the atmospheric neutrino mixing. New 7 strings will have a vertical spacing of approximately $2 \mathrm{~m}$ and will be deployed inside the DeepCore volume approximately $20 \mathrm{~m}$ apart from each other. New optical modules will use a multi-PMT design resulting in an increase of the effective photocathode area. The IceCube Upgrade will also include new calibration devices, which are expected to provide better knowledge about the ice properties, as well as calibrate the new and currently existing DOMs. The detector will have an energy threshold of a few $\mathrm{GeV}$ and is expected to have a few percent sensitivity to the atmospheric mixing parameters. The IceCube Upgrade received seed funding in September of 2018 and is expected to be deployed in 2023.

\section{References}

[1] ICECUBE collaboration, M. Aartsen et al., The IceCube Neutrino Observatory: Instrumentation and Online Systems, JINST 12 (2017) P03012, [arXiv: 1612 . 05093].

[2] ICECUBE collaboration, R. Abbasi et al., The design and performance of IceCube DeepCore, Astropart. Phys. 35 (2012) 615 - 624, [arXiv:1109.6096].

[3] ICECUBE collaboration, M. G. Aartsen et al., Measurement of Atmospheric Neutrino Oscillations at 6-56 GeV with IceCube DeepCore, Phys. Rev. Lett. 120 (2018) 071801, [arXiv: 1707. 07081].

[4] P. Eller, F. Hunag and M. Larson, Measurement of Atmospheric Tau Neutrino Appearance with IceCube/DeepCore, XXVIII International Conference on Neutrino Physics and Astrophysics (Neutrino 2018).

[5] K. N. Abazajian et al., Light Sterile Neutrinos: A White Paper, arXiv:1204.5379.

[6] ICECUBE collaboration, M. G. Aartsen et al., Searches for Sterile Neutrinos with the IceCube Detector, Phys. Rev. Lett. 117 (2016) 071801, [arXiv:1605.01990].

[7] ICECUBE collaboration, M. G. Aartsen et al., Search for sterile neutrino mixing using three years of IceCube DeepCore data, Phys. Rev. D95 (2017) 112002, [arXiv:1702 .05160].

[8] M. Leuermann, Results from Testing the Neutrino Mass Ordering with Three Years of IceCube DeepCore Data, XXVIII International Conference on Neutrino Physics and Astrophysics (Neutrino 2018) .

[9] ICECUBE collaboration, M. Aartsen et al., Search for Nonstandard Neutrino Interactions with IceCube DeepCore, Phys. Rev. D97 (2018) 072009, [arXiv: 1709.07079 ]. 